\title{
P-0449
}

\section{THE HUNGER GWWES;}

\section{TASTE, GLP-1 RND FEMSLE GENDER INYOLVED}

\author{
Baretić Maja ${ }^{1}$, Kušec Vesna ${ }^{2}$, Uroić Valentina ${ }^{3}$, Pavlić-Renar Ivana $^{1}$, Altabas Velimir ${ }^{4}$

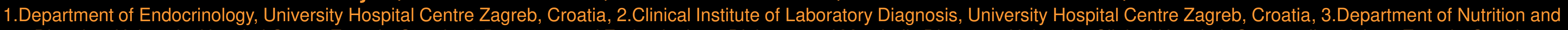 \\ Dietetics, University Hospital Centre Zagreb, Croatia, 4.Department of Endocrinology, Diabetes and Metabolic Diseases, University Clinical Hospital "Sestre milosrdnice", Zagreb, Croatia
}

BACKGROUND: The incretin hormone glucagon-like peptide-1 (GLP-1) is released from intestinal enteroendocrine $L$ cells in response to a carbohydrate meal. GLP-1 has many levels of action, but currently it is primarily known for its effect on beta cells. GLP-1 reduces gastric emptying, lowers appetite by promoting satiety via hypothalamic receptors and reduces food intake and leads to weight loss. The gastrointestinal tract is the major connector between food and body weight, it senses basic tastes in a similar manner as the tongue and through similar G-protein-coupled taste receptors. The link between gut hormones and eating behavior is well known, though it has not been thoroughly explored. There is no specific group of diabetic (or non-diabetic) patients identified as GLP-1 receptor agonist "responders" or "non-responders" with respect to weight loss. Some patients report a diminished craving for food following GLP-1 therapy and a change in food preference.

AlMS: The aim of this study was to explore how GLP-1 influences taste preference in different genders, insulin sensitivity and body composure.

METHODS: Effects of GLP-1 infusion on taste preference were investigated in 14 healthy participants $(6$ males, 8 females) in a double-blind, placebo-controlled crossover study. Insulin sensitivity was estimated with simultaneously measured fasting plasma glucose and insulin, body composition was estimated with bioelectrical impedance analysis (total fat mass, mean fat percentage). After an overnight fast and an oral sodium load delivered in the form of soup, participants received placebo $(500 \mathrm{ml}$ of $0.9 \%$ saline) over a 3-hour period. At the end of infusion, participants chose their current food preferences from illustrations of food types representing 5 tastes (sweet, bitter, salty, sour, and umami). After 7 days, the participants received an infusion of synthetic GLP-1 (1.5 pmol/ $\mathrm{kg} / \mathrm{min}$, dissolved in $500 \mathrm{ml}$ of $0.9 \%$ saline) and reported their food preferences again.

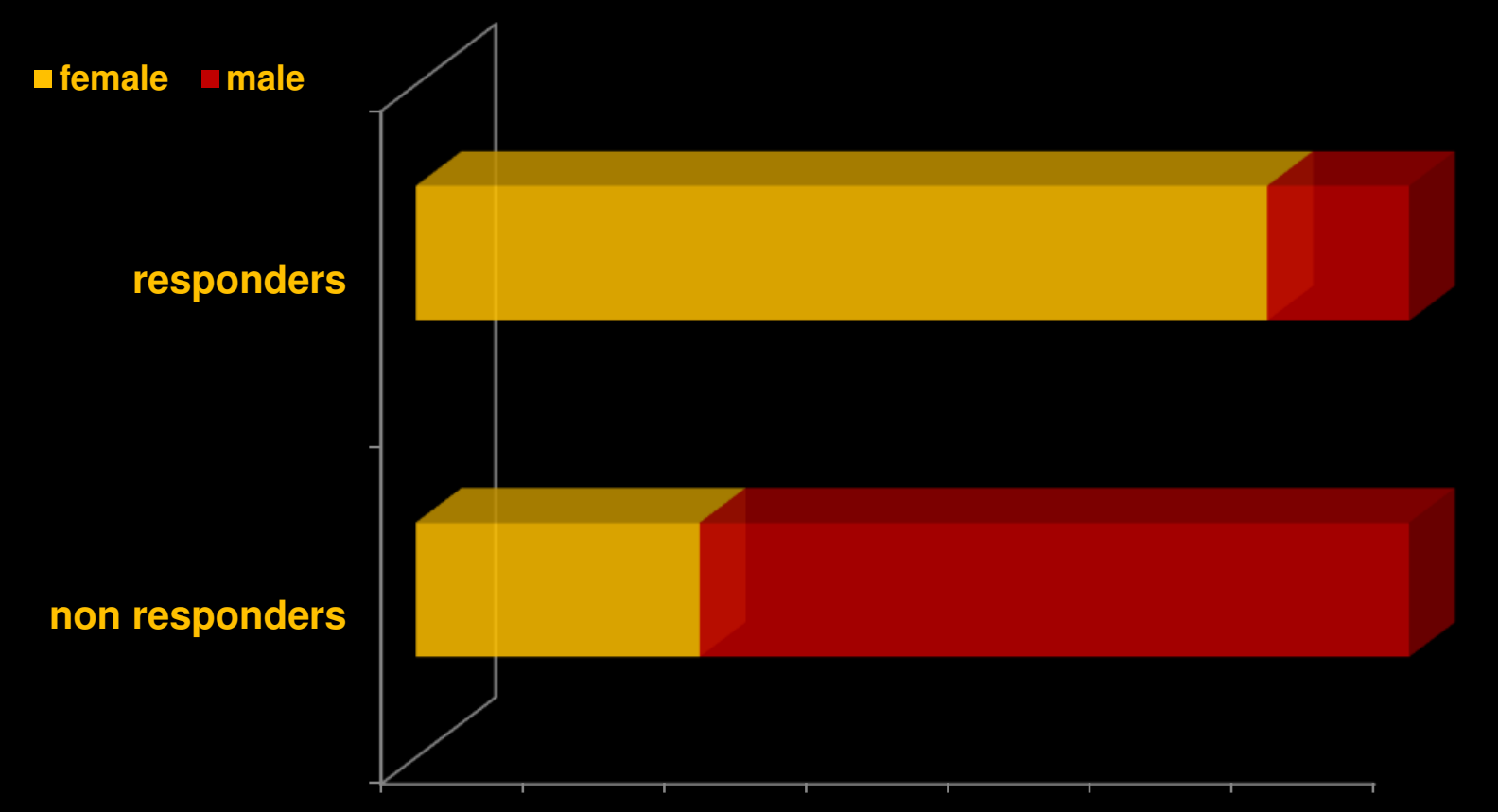

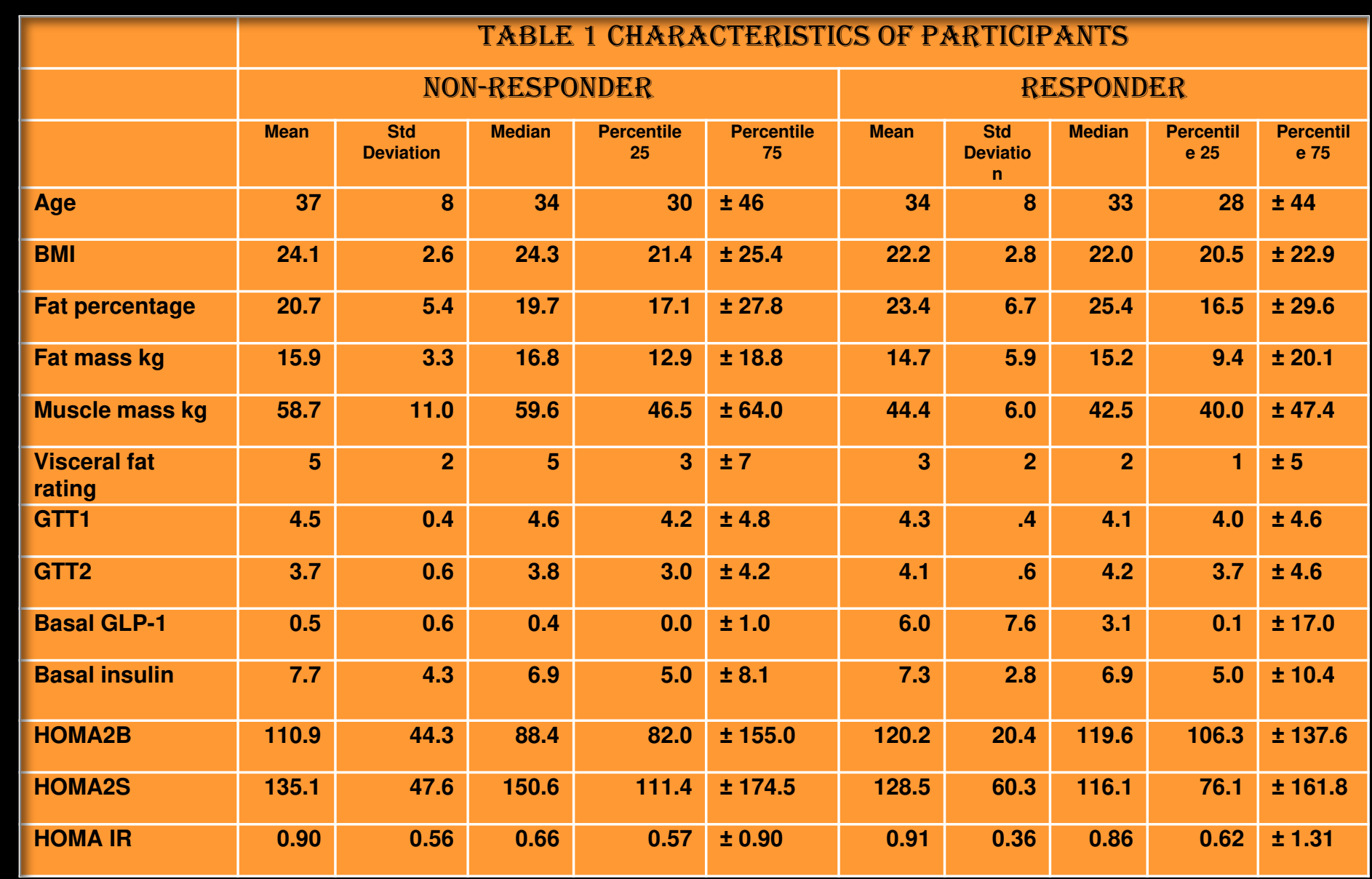

RESULTS: Change of taste preference after GLP-1 infusion but not after placebo was reported as response, and non-response was reported in the case of taste persistence. A statistically significant difference in response type was found between sexes $\left(x^{2}=4.667, \mathrm{df}=1, \mathrm{p}=0.031\right)$, with women being more likely to change their taste preference after GLP-1 infusion than men. There was a positive correlation in responders between the body composition parameter (body fat mass in kg) and basal insulin levels after GLP-1 infusion. We also found a strong positive correlation between insulin resistance and insulin level after GLP-1 infusion in responders $(r=0.89 p<0.01)$

DISCUSSION: The results of this study indicated that healthy non-obese women were more likely to change their taste preference after GLP-1 infusion than men. Observed change of taste upon GLP-1 infusion in women might be ascribed to estrogen. The weight-lowering effects of estrogens are associated with decreased food intake and increased energy expenditure in experimental animals, modulating energy expenditure and feeding behavior through leptin-like effects in the hypothalamus. Estrogens act directly on brain serotonin neurons. The weight-lowering effects of estrogens are accomplished by receptor-mediated delivery of GLP-1. According to current findings and this study data, recently developed estrogen/GLP-1 medication may be a new strategy for obesity therapy. Also, a current obesity therapy, a GLP-1 receptor agonist, may be more effective (or applied in lower dosages) in females. Gender differences in the physiology of eating and response to GLP-1 stimuli may contribute to better understanding of human obesity and eating disorders. For patients with more visceral fat and higher insulin resistance, a GLP-1 receptor agonist could be more effective (or applied in lower dosages) for the treatment of diabetes.

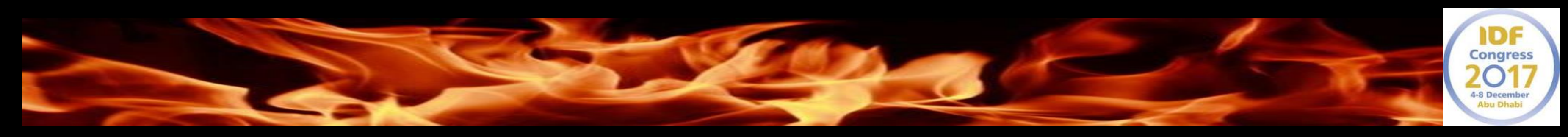

